\title{
Negative Ion Mode Evolution of Potential Buildup and Mapping of Potential Gradients within the Electrospray Emitter
}

\author{
Boguslaw P. Pozniak and Richard B. Cole \\ Department of Chemistry, University of New Orleans, New Orleans, Louisiana, USA
}

Differential electrospray emitter potential (DEEP) maps, displaying variations in potential in the electrospray (ES) capillary and in the Taylor cone, have been generated in the negative ion mode of ES operation. In all examples, measured potential was found to be the highest at the points furthest into the Taylor cone, and values descended to zero at distances beyond approximately $15 \mathrm{~mm}$ within the ES capillary. In agreement with results obtained previously in the positive ion mode, negative mode data show a strong influence of electrolyte concentration on measured potentials. Weakly conductive solutions exhibited the highest values, and the steepest gradients, at points furthest into the Taylor cone. However, these same low conductivity solutions did not yield nonzero measured potentials to as deep a distance into the ES capillary as was possible from their higher conductivity counterparts. Addition of a readily reducible compound lowered measured potentials at all points near the ES capillary exit, in accordance with the description of the ES device as a controlled-current electrolytic cell. The development of potential inside the ES capillary upon the onset of ES was also studied, and initial results are presented. Potential waves are observed that can require $15 \mathrm{~min}$ or longer, to stabilize. The slow drift to steady potentials is evidence of upstream movement of electrochemically-produced species and follow-up reaction products; low conductivity solutions require longer intervals to reach a steady state. Potentials measured along the central ES axis reflect those at the ES capillary surface, although equipotential lines can be considered to be more compressed at the latter surface. (J Am Soc Mass Spectrom 2004, 15, 1737-1747) () 2004 American Society for Mass Spectrometry

$\mathrm{T}$ The phenomenon of electrospray (ES), since its early invention, has found many applications in an astonishing variety of scientific and technical disciplines [1]. In chemical analyses, it greatly increased the ability of researchers to obtain gas-phase ions directly from solutions. This revolutionized the analysis of large biopolymers. Many practitioners, however, use electrospray without paying attention to the underlying physics of the process. In many instances, this approach is justified, but in others, principles must be more thoroughly understood if one seeks to extend existing applications.

The fact that electrochemical processes must participate in the sustained electrospraying of liquids was recognized many years ago [1]. Later it was established that the electrospray apparatus is not far different from a regular electrochemical cell, and the vocabulary of electrochemistry was used to describe its operation [2-4]. It was proposed and experimentally proven that

Published online October 28, 2004

Address reprint requests to Dr. R. B. Cole, Department of Chemistry, University of New Orleans, 2000 Lakeshore Drive, New Orleans, LA 70148, USA. E-mail: rcole@uno.edu the ES device operates as a controlled-current electrolytic cell $[5,6]$. Counting ES approaches among them, there have been many successful reports of the combination of on-line electrochemistry and mass spectrometry to analyze electrochemical products, including the utilization of electrochemical transformations to ionize neutral products, thereby rendering them amenable to mass spectral analyses [7-14].

Electrospray, when used as an ion source in mass spectrometry, creates droplets containing an excess of positive charge (positive ion mode) or negative charge (negative mode). In order to provide current to support electrospray ionization, an electrochemical reaction must take place at a conductive surface in the device, most often at a metal capillary. Products of electrochemical reaction leave the metal/solution interface and travel to the bulk solution inside the capillary. Current density at the surface, and by this, the yield of products, will be different for each position at the capillary surface which experiences a different interfacial potential [15-18]. Due to the axial symmetry of the capillary, it is sufficient to recognize only two coordinates: axial and radial. Total electrospray current is a sum (more precisely an integral over the surface) of local current densities. Total current is a parameter 


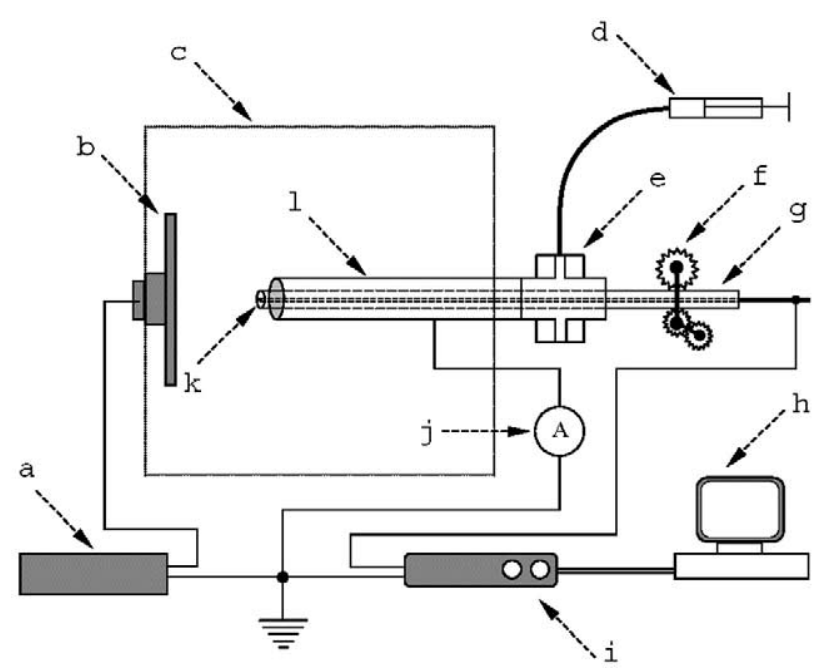

Figure 1. Diagram of the experimental device used to make potential measurements. (a) high voltage power supply; (b) brass plate serving as ES counter-electrode; (c) Faraday cage; (d) syringe pump; (e) cross union; (f) mechanical positioning device for wire electrode assembly; (g) fused silica capillary; (h) computer workstation; (i) potentiostat; (j) ammeter; (k) Pt wire electrode assembly sealed in fused silica exposing a disk-like surface; (1) Pt ES capillary.

externally imposed on the system, whereas the local distribution of potential is a system response. Local current density and local potential are interrelated through electrode kinetics considerations. The local potential will distribute itself such that the sum of locally produced currents will constitute a minimum of energy dissipation [15-17].

This article presents measured differential electrospray emitter potential (DEEP) maps acquired in the negative ion mode of electrospray. The goal was to measure how different solution parameters of the electrosprayed liquid affect the distribution of the potential. This work is an extension of the previous work done in the positive ion mode [19], and it adds experimental data to an area where only computational simulations of potentials in electrospray existed previously $[3,18]$. Due to accumulated expertise and improvement in the experimental set-up, the data are even more precise than those acquired for the positive ion mode [19]. In addition, the temporal component of potential build-up in the ES capillary was also investigated at various positions along the ES capillary.

\section{Experimental}

\section{Apparatus}

Experiments were performed using an ES system modified by the addition of a central moveable wire probe electrode within the ES capillary (Figure 1, built inhouse and described previously [19]). The external 99.95\% Pt ES capillary (Goodfellow Cambridge Ltd., Huntington, England, UK) has an i.d. $=0.5 \mathrm{~mm}$, and an o.d. $=0.6 \mathrm{~mm}$, whereas a platinum wire $(99.9 \%$, Alfa Aesar, Ward Hill, MA), $0.127 \mathrm{~mm}$ in diameter, sur- rounded by a fused silica tube (o.d. $=0.32 \mathrm{~mm}$, SGE Inc., Austin, TX), constitutes the mobile probe electrode placed inside the capillary. This wire probe is isolated so that only the tip of the Pt is exposed (having the form of a disk) and in electrical contact with the solution. The position of the wire probe with respect to the end of the capillary is now controlled mechanically. The total ES current is measured using an ammeter in series with the ES capillary. The open circuit potential (OCP) is measured between the internal wire probe and the ES capillary under open circuit conditions (high impedance, minimal current between electrodes).

Electrospray high voltage was provided by a high voltage supply (Glassman, High Bridge, NJ). The counter-electrode was comprised of a brass plate positioned at $6.0 \mathrm{~mm}$ from the end of the ES capillary. The wiring is such that the ES capillary is grounded and the brass counter-electrode is at high potential. For negative ion mode work, the latter is at high positive potential. Typical voltages used were between 2500 and 2800 volts. This relatively low setting made the Taylor cone "relaxed" with a full cone angle less than $60^{\circ}$. All experiments were performed in the single cone jet mode (Cloupeau classification [20]) and were done under protective atmosphere of nitrogen; however, solutions were not de-aerated. Recently, we found that ambient oxygen enters electrochemical reaction pathways and greatly changes the potential profiles. An upcoming publication will describe this effect [21].

Electrochemical data were acquired with a 660A electrochemical work station ( $\mathrm{CH}$ Instruments, Austin, TX). The instrument has an input impedance of $10^{12} \Omega$. Data points were collected every $0.1 \mathrm{~s}$. Data displayed in Figures 2, 3, and 4 are smoothed with a 24-adjacent average routine. For potential measurements, the reference electrode port of the workstation was clipped to the wire electrode probe, whereas the working electrode port on the workstation was grounded. This configuration was necessitated by the design of the potentiostat which has a ground connection to the working electrode port. The same ground is connected to the ES capillary, and the potential difference is measured between the ground and the reference electrode port. Due to the convention that the working electrode potential is measured with respect to a reference electrode, in our wiring, the sign of the reading was reversed, and this was rectified in all pertinent figures. In the negative ion mode, the wire electrode potential is higher than the ES capillary potential. Of course, it would be improper to assume that the ground has a constant potential or that it can serve as a reliable reference electrode.

\section{Probe Electrode Preparation}

All experiments were performed with the same wire probe electrode. A slightly modified electrode was used in this experimental series compared to those described previously [19]. At the very end of the wire, a smaller 

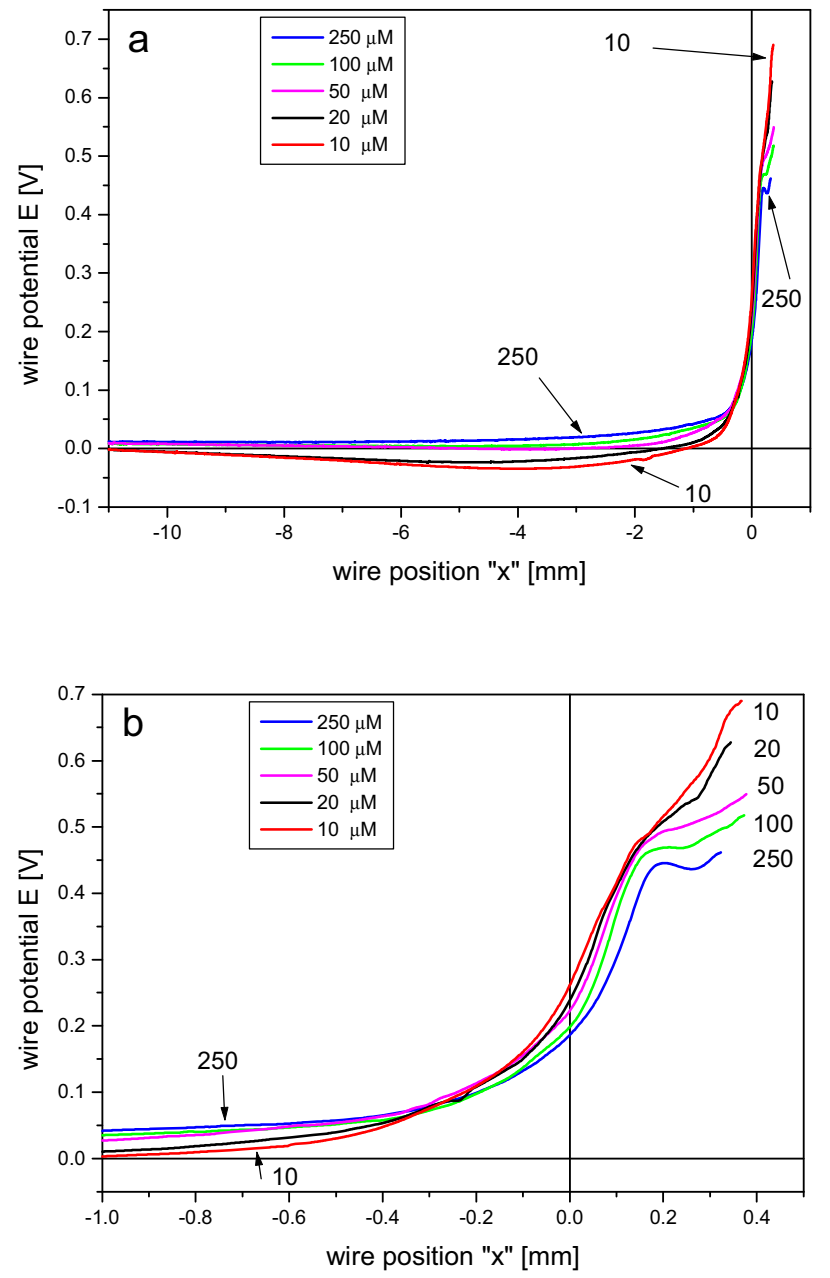

Figure 2. DEEP maps acquired from solutions comprised of $100 \%$ methanol with added supporting electrolyte $(\mathrm{KCl})$ present at concentrations of: 10, 20, 50, 100, and $250 \mu \mathrm{M}$. (a) Full range of wire probe positions; (b) close-up of region near the ES capillary exit. The highest potential at $\mathrm{x}=0$ corresponds to the map for 10 $\mu \mathrm{M}$ concentration, the next lower for $20 \mu \mathrm{M}$, and so on.

fused silica tube was inserted into a larger one, and the polyimide isolation was burned off of the exterior of the innermost fused silica. Afterwards, the last centimeter of the wire probe electrode (i.e., the platinum wire covered by silica isolation such that only the disk-like end of the wire is exposed) had an outside diameter of less then 0.20 $\mathrm{mm}$ compared to $0.32 \mathrm{~mm}$ previously. This construction imposes less disturbance to liquid flow inside the capillary, and less shielding of the internal surface of the ES capillary from the influence of the counter-electrode. Before sealing, the tip of the wire was flame annealed. This procedure made it smoother and cleaner compared to previously used sandpapered tips.

\section{Differential Electrospray Emitter Potential (DEEP) Map Acquisition}

After launching ES, time is allowed for the system to reach a stable potential distribution, typically about
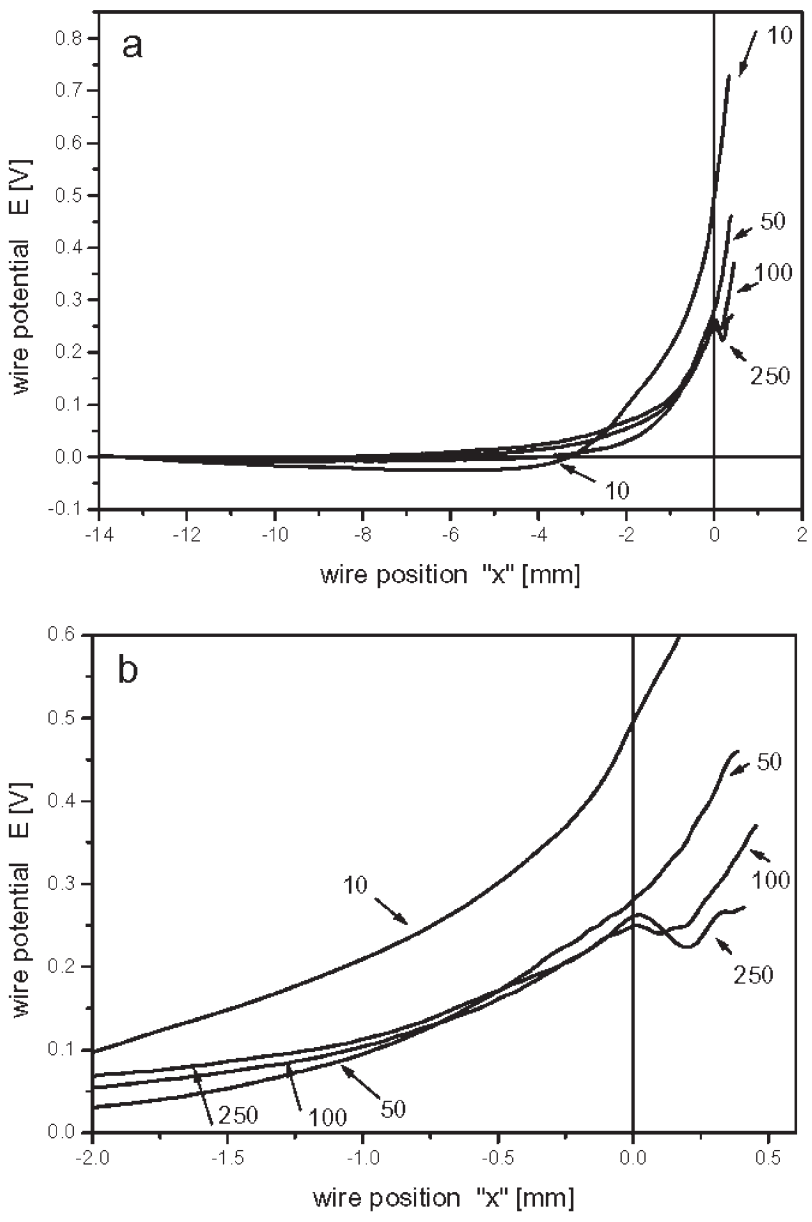

Figure 3. DEEP maps obtained from methanol : chloroform (90:10), with added $\mathrm{KCl}$ as supporting electrolyte at concentrations of: 10, 50, 100, and $250 \mu \mathrm{M}$. (a) Full range; (b) region of ES capillary exit.

$800 \mathrm{~s}$; then the wire is placed at a positive value of $\mathrm{x}$ $(+0.35 \mathrm{~mm})$. In a DEEP mapping experiment, the wire is mechanically drawn out the back end of the capillary at a constant velocity of $10 \mu \mathrm{m} / \mathrm{s}$ ( $1 \mathrm{~mm}$ per $100 \mathrm{~s})$. This velocity is chosen to minimize solution perturbations inside the capillary, but at the same time, allow one to complete the experiment in a reasonable time period without having to refill the syringe. Use of lower velocities, or slightly higher ones, does not alter the obtained results. All experiments were performed at a flow rate of $200 \mu \mathrm{L} / \mathrm{h}$; one syringe fill $(>133 \mu \mathrm{L})$ lasts about $2400 \mathrm{~s}$. Because the act of moving the wire probe backwards into the capillary liberates some volume at the probe tip, such backward probe movement somewhat reduces the flow rate of the liquid to the capillary exit. A calculation based on the wire isolating tube o.d. $(0.32 \mathrm{~mm})$ and the velocity of the probe movement, shows that this reduction represents less than $2 \%$ of the flow, so this error was ignored.

The position of the tip of the wire probe, as it protrudes from the ES capillary, was observed through an optical microscope in order to set the zero point on 

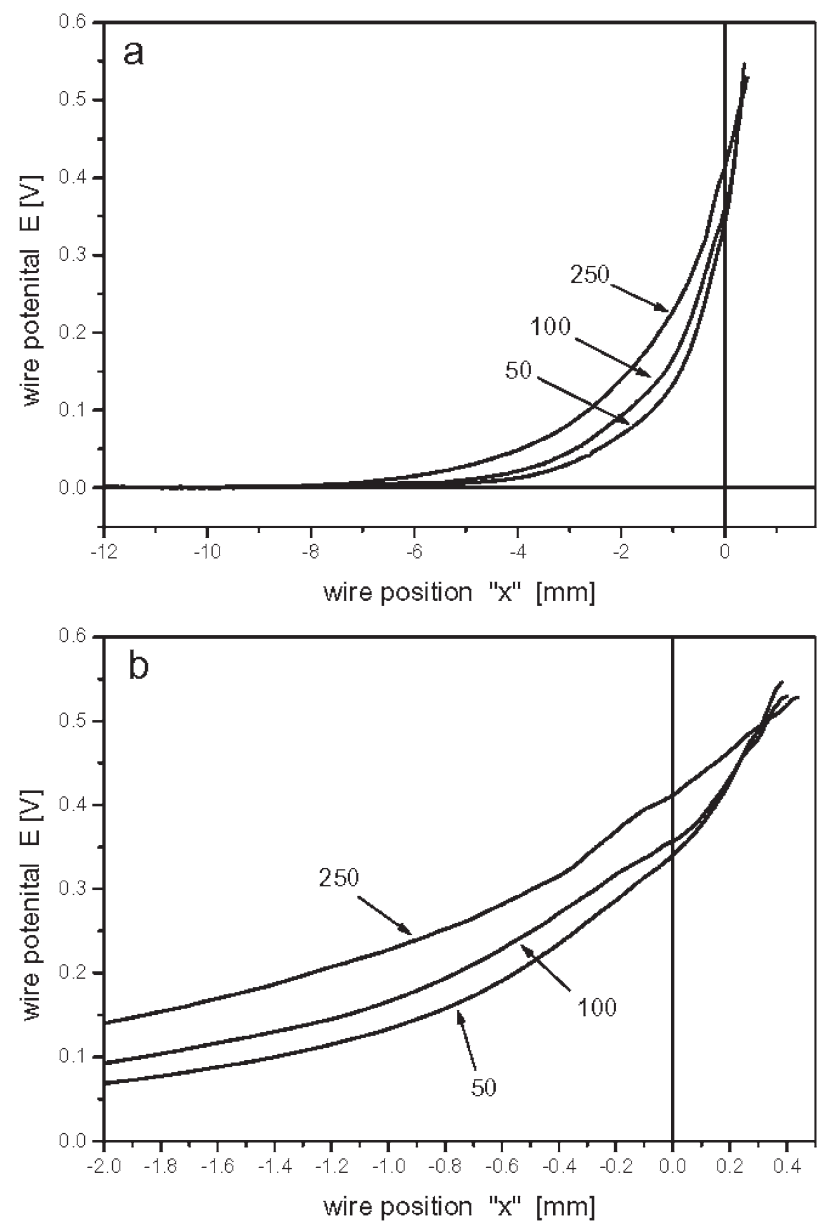

Figure 4. DEEP maps acquired from a 1:1 mixture of methanol and chloroform with added $\mathrm{KCl}$ at concentrations of: 50, 100, and $250 \mu \mathrm{M}$. (a) Full range of " $\mathrm{x}$ " values; (b) region nearest to the ES capillary exit.

the $x$-axis scale. Our coordinate system was chosen such that value of zero $(x=0)$ corresponds to the point where the wire is flush with the capillary exit. All other positions are read from the positioner: positive values correspond to cases where the wire protrudes out of the capillary and enters inside the base of the Taylor cone whenever it is formed; negative values indicate that the wire is withdrawn inside the ES capillary.

\section{Open Circuit Potential Versus Time Measurements}

The wire electrode is placed at a fixed position $(x=0$, flush with the capillary exit) and the open circuit potential difference between the wire and the capillary is recorded as a function of time. An extension of this experiment is one in which the wire is held static at various positions of $\mathrm{x}$ while $\mathrm{OCP}$ is measured over time.

\section{Chemicals}

Solvents were used as purchased, without further purification. Methanol was provided by EMD Chem- icals (Gibbstown, NJ), chloroform by J. T. Baker Inc. (Philipsburg, NJ), potassium chloride by Mallinckrodt (St. Louis, MO), and 1,4-benzoquinone and lithium trifluoromethylsulfonate $\left(\mathrm{LiCF}_{3} \mathrm{SO}_{3}\right)$ by SigmaAldrich (St. Louis, MO). Information pertaining to the electrochemistry of employed solutions appears in Appendix I.

\section{Results and Discussion}

\section{Negative Ion Mode DEEP Maps as a Function of Supporting Electrolyte Concentration}

Methanol solvent. Differential electrospray electrode potential (DEEP) maps were obtained for a series of solutions of methanol containing varying concentrations of supporting electrolyte: 10, 20, 50, 100, and $250 \mu \mathrm{M} \mathrm{KCl}$ (Figure 2). These DEEP maps reveal potential changes as a function of wire probe position within the ES capillary. The least concentrated solution $(10 \mu \mathrm{M})$ yields the highest measured potential at $\mathrm{x}=0$, but the values also decay the fastest with movement into the ES capillary. As the electrolyte concentration is raised, the maximum potential recorded at the most positive $x$-values becomes progressively lower. Additionally, it is noted that the higher conductivity solutions give nonzero values to positions deeper within the ES capillary relative to the less conductive solutions. All of the curves display similar values near $\mathrm{x}=-0.3 \mathrm{~mm}$ (a crossing point).

Organic solvents are characterized by low charge carrier concentrations and, because of this, high specific resistivities; hence, ohmic potential drop in such liquids is large. Liquid specific conductivity is also a linear function (in the first approximation, but correct enough) of concentration of ions. A solution of the lowest concentration of supporting electrolyte will set the highest ohmic drop. This would lead to the highest potential at the capillary exit and the fastest drop off upon movement away from the counter-electrode. The solution exhibiting the lowest ohmic drop (highest conductivity) would yield the lowest potential at the ES capillary exit, but also exhibit the shallowest potential gradient upon moving inside the ES capillary. Such a progression is nicely demonstrated in Figures 2,3 , and 4 . These figures are very much the mirror images of the previously presented study of acetonitrile in the positive ion mode [19]. Here, due to improvements in precision of the acquired data, the trends are quite pronounced.

In generating DEEP maps, it must be kept in mind that there are two basic sources of the measured potential of metals in solution [22-24]: faradaic exchange of electrons between species in the vicinity of the metal (taking into account required overpotentials), and nonfaradaic contributions, i.e., dielectric orientation of molecules and specific adsorption at the metal surface. In order to establish a true equilibrium potential at the metal (Galvani potential), in the thermodynamic sense, 
there must be a reversible redox couple at the electrode surface. In the absence of such a couple, a conservative viewpoint will contend that the potential is not defined, which in turn leads to trouble in defining an overpotential $[24,25]$. To further complicate matters, when current passage progresses, reaction products are accumulated at the surface. Because they diffuse out at a rate slower than that of production, the opportunity exists for a new redox couple to be created and further contribute to the measured potential. Our probe wire electrode passes a negligible amount of current, and its behavior is close to that of an ideally non-polarized electrode. But products of electrochemical activity at the ES capillary wall arrive in its vicinity, inducing potential changes that alter the potentials initially set. So the final recorded potential difference (between the two platinum electrodes) is a result of a combination of electrode kinetics factors, including concentration polarization and activation polarization of the ES capillary emitter, and specific adsorption on both electrodes.

An additional factor influences open circuit potential (OCP) measurements, i.e., ohmic drop [22-25] due to resistance of the electrolyte between the probe wire electrode and the ES capillary. Furthermore, ohmic drop itself depends on two factors-specific resistivity of the electrolyte, and the geometry of the system. Organic liquids have high specific resistivities, but addition of large amounts of supporting electrolyte to reduce solution resistance is not practical in ES-MS because high levels of salt degrade performance by suppressing analyte ionization [26, 27]. For small electrodes (microelectrodes) the total resistance of the cell depends practically on the electrode surface area. In our system, a reasonable estimate of the ohmic resistance of the electrolytic cell made of the probe wire electrode and the ES capillary is in gigaohms for pure methanol, and megaohms when $10 \mu \mathrm{M} \mathrm{KCl}$ is present (see Appendix II). For such high resistivities, even picoampere current drawn by a potentiostat will produce a noticeable ohmic drop. Moreover, during our measurements, the resistivity of the cell is not constant over time. Changes in specific conductivity can be attributed to changes in liquid composition, due to ion production at the ES capillary surface, and to ion migration in the electric field. What is more important for a comparative study such as ours, however, is that the ohmic drop is not constant in runs employing different concentrations of supporting electrolyte. Let a typical electrospray current be $100 \mathrm{nA}$, and let the liquid flow be $200 \mathrm{~mL}$ per $\mathrm{h}$; this amount of current produces about $1 \mathrm{pmol} / \mathrm{s}$ of ions. When combined with flow rate considerations, this production can increase downstream ion concentration by almost $2 \times 10^{-5} \mathrm{M}$. For a pure solvent, and for a solvent with a low concentration of supporting electrolyte, such changes are significant.

In a blank experiment, where no high voltage was applied to initiate ES, when the wire probe electrode was moved, there were no significant changes of potential, that is, the measured potential difference gave a value close to zero $( \pm 20-30 \mathrm{mV}$ depending on the solution). However, this type of blank is not fully informative because it cannot be performed for positive $x$ values, as without ES, of course, there is no Taylor cone. If ES is performed in such a way as to let the wire probe puncture the edges of the Taylor cone, the spray emanates only from the tip of the wire, and the measured potential jumps immediately to very high values, e.g., 5-9 V. In puncturing the Taylor cone, additional equipotential lines are crossed by the probe wire, but conductivity along the edges of the Taylor cone may be higher than conductivity through the bulk solution. When the wire probe is within the bulk, the problem of lowered electrical contact exists for all potential measurements at positive $x$ values. A conclusion here is that changes in ohmic drop over time may influence results obtained for OCP measurements, and the DEEP maps presented in this work were not corrected for such effects.

Methanol/chloroform mixtures. For a (90:10) methanol: chloroform mixture, DEEP maps are shown in Figure 3, whereas those obtained from a (50:50) mixture are shown in Figure 4. As was the case for methanol solutions devoid of chloroform (Figure 2), at the most positive $x$-values, the lowest conductivity solutions exhibit the highest measured potentials, whereas progressively higher conductivity solutions gave steadily decreasing values. Moreover, the DEEP maps exhibit crossing points in both the 50:50 mixture (at positive values of $x$ ) and in the 90:10 mixture, indicating that solutions of progressively higher conductivity allow non-zero measured potentials at increasing distances into the ES capillary. There is no fundamental reason why these DEEP maps should exhibit crossing at a single point. In our interpretation of these maps as reflecting (but not being equal to) interfacial potential, a consistency of electrochemical parameters is achieved by checking the area below the DEEP map. Current density is a function of local potential through a ButlerVolmer type relationship. Integration of current density over the whole current producing surface will give total current to the capillary (to have a quantitative value, more mathematical refinement is necessary to account for the distortion between measured values at the capillary central axis and the potential at the metalsolution interface, the exponential relation between potential and current, and a knowledge of the details of the surface reaction $\{s\}$ is also required). The integrated area should grow with supporting electrolyte concentration because the total current has increased, but the factors that determine the precise curvature of the DEEP map are not intuitively obvious. In fact, in the simplest model in which the only factor determining the potential is the solution resistance, the DEEP map curvature is a function of resistivity, while the "offset" above the zero line is a function of the total current. So a change in supporting electrolyte can both vertically shift and bend the curve independently, to determine a 
crossing point. The above conclusion is a safe one to make only when the difference between solutions is simply the concentration of the supporting electrolyte. Pure solutions may exhibit different electrochemistry in the absence of supporting electrolyte, and their potential gradients may not correspond to direct extrapolations to zero concentration of the added salt.

DEEP maps also show unexpected dips at high positive values (Figures 2 and 3), i.e., for $x$ values greater than $0.2 \mathrm{~mm}$. The effect is the largest for solutions of highest salt concentration. Due to geometrical bulkiness, when the working electrode probe protrudes further into the Taylor cone (positive values of $x)$, potential measurements exhibit a larger error. Potential gradients in the volume inside the Taylor cone are very steep. Regretfully, the bulky wire electrode cannot be perfectly centered. As it enters into the Taylor cone, it crosses equipotential lines that have both axial and radial components, and the electrode area has become large compared to the potential gradient. Thus, different parts of the wire probe will be at different potentials, and the resulting value for the measured potential is a combination of many factors.

In the numerical modeling by Van Berkel and coworkers [18], it was shown that all the current is produced from a region not extending deeper than a 3:1 aspect ratio of the capillary diameter. For our capillary, that would mean that the first $1.5 \mathrm{~mm}$ will produce all the current. For a real capillary an additional problem arises: a real non-beveled cut capillary has a finite wall thickness, which produces a wettable front surface facing the counter-electrode. That surface is particularly exposed to the surrounding atmosphere and it is the metal point closest to the tip of the Taylor cone. It is thus in excellent electrical contact with the surface of the Taylor cone that is charged and may have a lower resistance than the bulk solution deeper inside the cone. It is very likely that this front metallic surface is the site of most of the electrochemical work. As we plot our DEEP maps along the capillary axis we presume that the potential distribution measured this way is reflective of the potential variations along the capillary/ solution surface. However, the existence of the metallic surface constituting the cut end of the ES capillary causes the high potential within the capillary to be dissipated over a shorter distance into the capillary than would otherwise occur.

\section{Variations in Potential as Measured by a Static Probe at the Onset of Electrospray}

Methanol solvent. Development of potential over time upon initiation of ES, employing a fixed position of the wire probe electrode, is shown in Figure 5 for a wire position of $x=0$ (probe flush with ES capillary exit). First, after switching on the ES high voltage, the measured potential rises rapidly for about $1 \mathrm{~s}$, due to capacitative charging. Then, in a matter of tens of

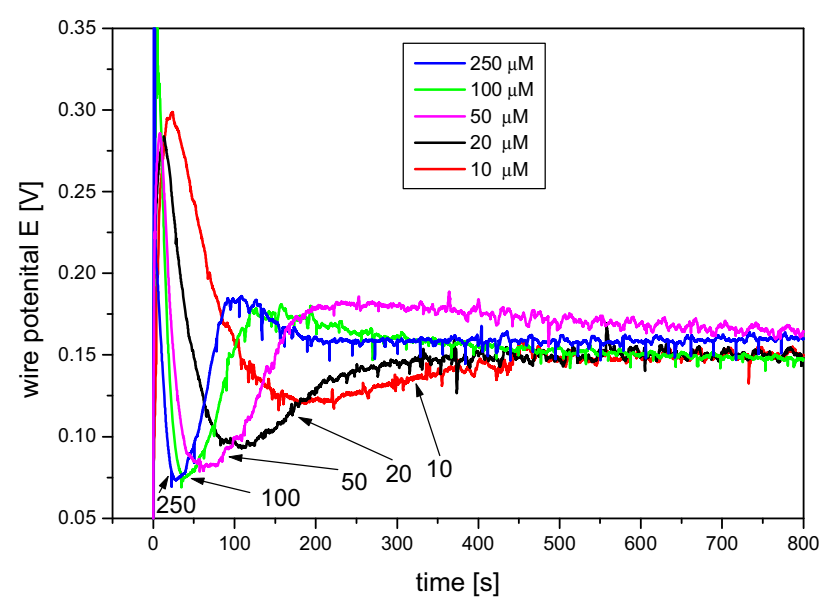

Figure 5. Evolving measured potential difference between wire probe and grounded ES capillary as a function of time. The wire was stationed at a fixed position $x=0$. Time " 0 " corresponds to the application of high voltage to the counter-electrode. Curves correspond to various methanol (100\%) solutions containing different amounts of supporting electrolyte $(\mathrm{KCl})$, i.e., 10, 20, 50, 100, and $250 \mu \mathrm{M}$.

seconds, with the Taylor cone and filament jet fully formed, the measured potential rises to a maximum value, next, falls at an almost equally fast pace, then, grows again to a value only a little above the final one, and from there, slowly decays. The measured potential stabilizes in the shortest time for the most conductive solutions, but concomitantly, the amplitude of initial fluctuations is the largest. Total current to the ES capillary also varies slightly over time (not shown). Initially, ES current grows and reaches a maximum value (which is only about $10 \%$ higher than the initial or final current value) at about $100 \mathrm{~s}$ (where potential shows a local minimum), and then slowly creeps to a steady value. In agreement with previous reports [2628], in our experiments, total ES current to the capillary shows a weak dependence on concentration of KCl. ES current has been reported to vary in proportion to the square root of electrical conductivity [28], and our experimental data, starting with $14 \mathrm{nA}$ for zero concentration and reaching $114 \mathrm{nA}$ for $250 \mu \mathrm{M} \mathrm{KCl}$, is in excellent agreement with a square root relationship.

The early increase in measured potential (e.g., first 30 s, Figure 5) may be due to movement of an oxidized species involved in a redox couple away from the probe electrode. This oxidant cannot be methanol, but possibly a contaminant, which could be in the form of dissolved oxygen. The higher the concentration of supporting electrolyte, the faster the final steady state is being achieved. This is likely to be as much due to the higher concentration of ions, as to the higher total current which produces reduced species in greater quantities. A very similar situation is observed for other studied solutions (see Figure 6). In all cases, the time needed to reach the steady state is shorter for more conductive solutions. Of course, a change in supporting electrolyte concentration alters the conductivity of the 

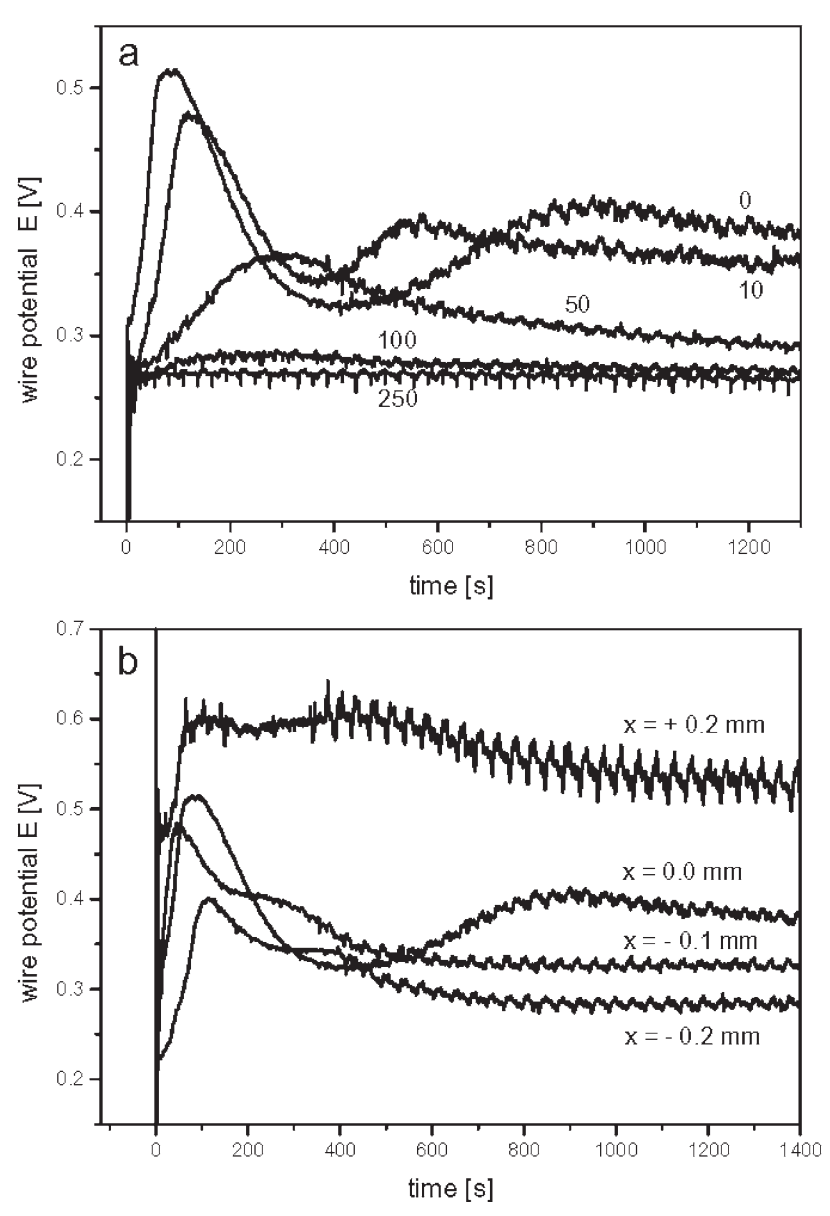

Figure 6. Evolving measured potential after onset of ES employing methanol:chloroform mixtures (90:10). (a) Development of potential versus time, with wire electrode position fixed at $\mathrm{x}=0$, for different concentrations $(\mu \mathrm{M})$ of supporting electrolyte, $\mathrm{KCl}$, as noted. (b) Four different positions of the probe wire electrode, as denoted in the figure, for (90:10) methanol:chloroform without $\mathrm{KCl}$.

solution. It is established that the ES device operates as a controlled current electrochemical cell $[5,6]$, meaning that the electrodes adopt the potential to maintain the demanded current. Careful measurement of the current shows that this picture is only a limiting one, that is, it is true only once a steady-state is achieved after initiation of the electrospray process. In the initial stages (e.g., the first three minutes), the current also shows fluctuations, as does the measured potential (Figure 5), although the former are smaller in relative value. Those initial stages are necessary for the electrode to produce initial quantities of ions and molecules which then distribute themselves in the cell, so as to balance existing forces. The higher the current, the faster the production, and finally, the shorter the time needed to achieve such a balance. The time required to achieve a steady state lengthens with decreasing conductivity of the solution, and may be quite long for solvents with little or no supporting electrolyte that exhibit low currents (e.g., 8-15 min, Figures 5 and 6). This reasoning may explain why solvents with low conductivities exhibit slow drifts in performance, which may also be manifested as a slow change in the intensity of a peak in a mass spectrum produced during ES-MS.

Despite recording large differences in measured potentials, and some differences in current values, no changes in the shape or size of the Taylor cone, or in the filament jet, were observed during the course of experiments. This reinforces the concept that electrospray is a "self-regulating" system where changes in the composition of the electrolyte, due to electrochemical reactions, are counterbalanced by changes in local potential at the interface, resulting in changes in local current densities.

Methanol/chloroform mixtures. A plot of the development of measured potential versus time for the wire probe electrode fixed at position $x=0$ in 90:10 methanol:chloroform is shown in Figure 6a. The solutions with either 0 or $10 \mu \mathrm{M}$ of $\mathrm{KCl}$ show large fluctuations in the measured potential, very much like those from "pure" methanol solvent (Figure 5). The next measured potential track for $50 \mu \mathrm{M}$ of $\mathrm{KCl}$ is fluctuating far less, and the potential-time profile for the highest concentration of $\mathrm{KCl}, 250 \mu \mathrm{M}$, shows a stable measured potential virtually from the outset.

To further explore potential "waves" in solvent mixtures, the wire probe electrode was fixed at four different positions in the 90:10 methanol:chloroform mixture, (Figure 6b). When the wire electrode is most exposed $(\mathrm{x}=+0.2 \mathrm{~mm}$, Figure $6 \mathrm{~b})$, the measured potential reaches a steady value fairly fast, i.e., in about $100 \mathrm{~s}$, but then a secondary wave comes in, and the value finally stabilizes in about $700 \mathrm{~s}$. The most variable is the potential-time profile obtained at the $\mathrm{x}=0$ position; it is the same data file as in the upper panel (no added $\mathrm{KCl}$ ). The measured potential tracks for the wire electrode in positions deeper inside the capillary appear as lower amplitude "echoes" of the potential-time profile for $x=0$. Figure $6 \mathrm{~b}$ is a good illustration of the existence of such concentration density waves of electrolysis products. Such waves appear to be most prominent where most of the current is produced (near position $x=0$ ), but they also exist deeper inside the capillary, and are apparently less variable closer to the tip of the Taylor cone where the balance of charges must be very strict in order to assure stable operation.

The existence of these potential waves serves as evidence that species produced by electrochemistry near the ES capillary exit can move upstream to points further within the capillary. In negative ion experiments, if these products of electrochemical reaction are cationic, they will undergo migratory motion in the upstream direction. Of course, all products and existing components of the solution are subject to diffusional motion caused by chemical potential gradients. The flow of liquid in the capillary adds an additional velocity component toward the capillary exit. Recently, it has been found from fundamental hydrodynamic considerations and by experiments [29-31] which find 
support from our own observations, that the hydrodynamic picture in the electrospray capillary with a Poiseuille-like laminar flow and a stagnant diffusion layer at the surface is overly simplified. As was noted by Fenn [32], "It does not take much imagination to realize that because of viscosity, the rapidly moving outer layer of [Taylor] cone liquid should be compensated in part by some upstream component of flow ...." The exact pattern of liquid flow is extremely difficult to assess. Ions of polarity opposite to the charge excess move in the direction opposing the flow of liquid, and they could overtake the flow if the electric field was high enough. A typical linear flow velocity in the employed capillary is $0.0283 \mathrm{~cm} / \mathrm{s}$; migration of common inorganic ions like potassium in methanol is $7.6 \times 10^{-4}\left[\mathrm{~cm}^{2} \mathrm{~s}^{-1} \mathrm{~V}^{-1}\right]$ [25]. Thus, a field strength of $37 \mathrm{~V} / \mathrm{cm}$ is sufficient to overtake the downstream flow (see Appendix III). While the slopes of obtained DEEP maps indicate maximum potential gradients approaching $10 \mathrm{~V} / \mathrm{cm}$, a combination of migration, diffusion, and turbulent flow can allow for upstream movement. Electrochemical sensing, as performed in this project, reflects the concentrations of chemical species in the vicinity of the wire probe electrode. Upstream movement will contribute to the supply of species to the metal surface of the electrochemical probe, and by this, will influence the obtained values, but in a fashion that evolves over time upon onset of ES.

A second real phenomenon observable in the potential-time profiles is that, after about $400 \mathrm{~s}$, a steady oscillation of measured potential develops at about $0.025 \mathrm{~Hz}$ (Figure 6b). The underlying processes responsible for this oscillation are not well understood. We propose that such oscillations may originate due to development of turbulent flow, or they may be the result of the oscillatory nature of surface reactions. Such oscillations in flow systems are fairly common and are well proven in theory and experiment [33]. Regardless of their exact origin(s), the observed oscillations may be amplified by liquid flow instabilities, however, the oscillations are not solely attributable to syringe pump imperfections. In our experiments, the stepping motor takes four steps per second. Furthermore, uneven flow caused by gas bubbles trapped in the connecting capillary, for example, immediately manifests itself as instability of the Taylor Cone, which may grow and contract in a type of "breathing" movement with large amplitude oscillations of potential and current. Over the course of experiments performed to generate Figures 5 and 6, potential oscillations were relatively small in amplitude, so any accompanying "breathing" of the Taylor cone was too subtle to observe with the naked eye. Notably, turbulences in flow patterns are not expected to be completely featureless; rather, they often have a periodicity associated with them.

The specific shapes of potential-time profiles are difficult to interpret without resorting to detailed numerical analysis of the electrochemical reactions at the surfaces, and concomitant chemical analysis of the
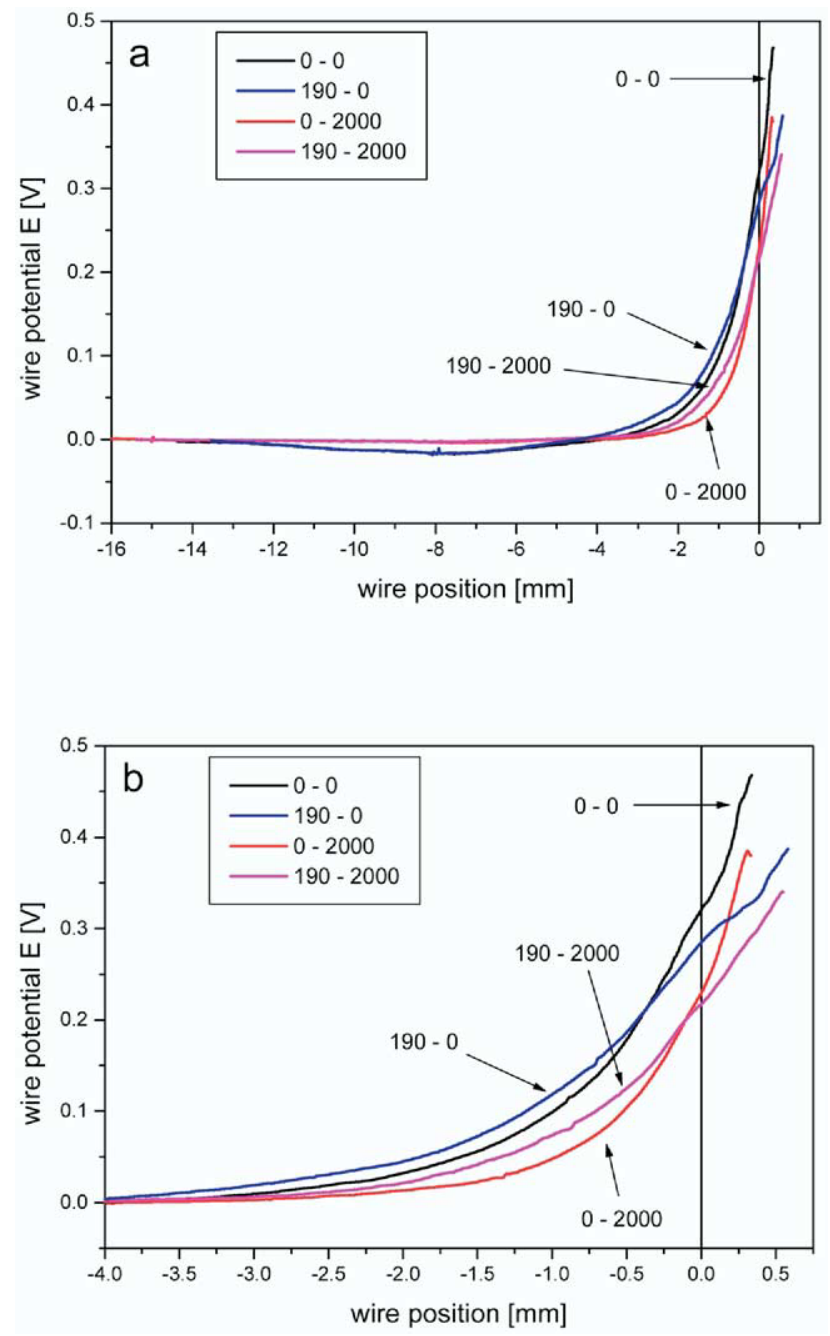

Figure 7. DEEP maps obtained for 1,4-benzoquinone in a mixture of methanol:chloroform (1:1). [0-0 (black)] indicates no supporting electrolyte, no 1,4-benzoquinone; [0-2000 (red)] corresponds to no supporting electrolyte with $2000 \mu \mathrm{M}$ 1,4-benzoquinone; [190-0 (blue)] indicates $190 \mu \mathrm{M}$ of $\mathrm{LiCF}_{3} \mathrm{SO}_{3}$ as supporting electrolyte but no 1,4-benzoquinone; [190-2000 (magenta)] denotes $190 \mu \mathrm{M}$ of $\mathrm{LiCF}_{3} \mathrm{SO}_{3}$ with $2000 \mu \mathrm{M}$ of 1,4-benzoquinone.

products, but they certainly prove that electrospray is an electrochemically evolving system that can require a substantial time to reach a steady state of operation.

\section{Alteration of Negative Ion Mode DEEP Maps Upon Addition of Easily Reducible Species}

1,4-Benzoquinone is an easily reducible species. Its halfwave polarographic reduction potential is significantly less than that of chloroform [34, 35] (see also Appendix I). Figure 7 shows acquired DEEP maps of methanol-chloroform (50:50) mixtures with added 1,4-benzoquinone (2000 $\mu \mathrm{M})$, and added supporting electrolyte $(190 \mu \mathrm{M}$ $\mathrm{LiCF}_{3} \mathrm{SO}_{3}$ ), with appropriate blanks. If there is no supporting electrolyte, addition of 1,4-benzoquinone visibly lowers the measured potential at all points close to the capillary exit (compare $0-0$ \{black\} with $0-2000$ \{red\}), but 
the two curves become indistinguishable at positions very deep inside the ES capillary. If the solution contains supporting electrolyte, again, addition of 1,4-benzoquinone lowers the measured potential at the points near to the ES capillary exit (compare 190-0 \{blue\} with 1902000). But again, both curves for 1,4-benzoquinone merge at distances more negative than $\mathrm{x}=-4 \mathrm{~mm}$. The presence of 1,4-benzoquinone does not change the total current to the capillary.

Clearly, the addition of 1,4-benzoquinone has a different effect on acquired DEEP maps than the addition of supporting electrolyte. 1,4-Benzoquinone will not dissociate in solution, so it will not directly alter solution conductivity in methanol and chloroform, and it will not increase the electrospray current. In the previous publication showing DEEP maps acquired in the positive ion mode, rubrene, an easily oxidizable species, was shown to substantially reduce the measured potential for all $x$ values [19]. The mirror image result in the negative ion mode presented here is less dramatic, but still convincing. In the positive ion mode, rubrene was particularly well chosen for illustrative purposes because the difference in oxidative potentials between rubrene and its solvent acetonitrile was large. In the negative mode, for the case of 1,4-benzoquinone in chloroform, the analogous difference is smaller, and therefore the expected drop in interfacial potential is less pronounced. 1,4-Benzoquinone thus reduces the interfacial potential in the current production region of the ES capillary because it can accept necessary electrons relatively easily. $2000 \mu \mathrm{M}$ 1,4-benzoquinone is a sufficiently high quantity to have all the electrospray current produced by reduction of 1,4-benzoquinone, if all of the compound could reach the capillary surface. Of course, 1,4-benzoquinone must diffuse to the electrode surface to accept charge. In an ES capillary with a constantly flowing liquid, the conversion factor is likely to be less than $100 \%$ [36], and part of the ES current may be supplied by the reduction of impurities and/or solvent, so the decrease of interfacial potential will be a function of flow velocity and total current.

\section{Conclusions}

Equipotential lines can be imagined as being more compressed at the capillary surface compared to the capillary center that is reported in DEEP maps. Thus, the derivative of the potential measured along the capillary axis is smaller (the potential gradient is less steep) than it would be if measured along a line very close to the capillary surface. Here, "very close" refers to distances just greater than a typical double layer. In other words, for positions near $x=0$, interfacial potential at the capillary surface may be a compressed map of the potential measured in this experiment. In a comparative sense, however, the progression of changes will be preserved as the concentration of supporting electrolyte changes.

Another conclusion can be made regarding accumu- lation of ions in the ES capillary that are of polarity opposite to those constituting the excess charge. It has been vividly demonstrated that the composition of electrolyte in the electrospray capillary is changing over time $[37,38]$. The electric field decays in strength as one moves further away from the counter-electrode, or toward the center of the ES capillary. It seems reasonable that ions could travel countercurrent until the velocities of field migration and liquid flow balance each other. Without a break in symmetry, they might be expected to accumulate in an equipotential cross section, forming a kind of a local maximum of concentration. The movement of ions may also be reinforced by hydrodynamic back flow and diffusion. In the final picture, in infinite time, there may be many of such concentration profiles of ions inside the cell, bearing a unique shape (and distribution) for each type of ion.

\section{Appendix I}

\section{Electrochemistry of Solvents and Dissolved Species}

Lithium or potassium ion is not being reduced in methanol; its redox potential is practically the same as in water (more cathodic than most other ions) [39, 40]. Chloride and trifluoromethylosulfonate anions obviously are not reducible. Moreover, methanol is difficult to reduce electrochemically; products will depend on the availability of water, $\mathrm{pH}$, and the catalytic properties of the surface; typically among the products is hydroxyl anion. The chloroform half-wave polarographic potential for reduction is $\mathrm{E}_{1 / 2}=-1.67 \mathrm{~V}$ versus SCE (in dioxane-water) [41]. It is reduced through a well-studied process called electrochemical dehalogenation. Formation of a radical anion is the first step, then, a neutral radical and chloride ion upon dissociation. Later, the fate of the radical depends largely on the availability of protons [41-43]. If they are available from, for example, amphiprotic methanol or traces of water, a second electron transfer is possible, and a neutral product joins the chloride ion as released products in an overall two electron per molecule process. The charge of the solution is, in half, obtained by reduction and release of chloride anion, and in the second half by reduction of a proton. The second step is thus $\mathrm{pH}$ dependent.

One possible product of chloroform reduction is methylene chloride, $\mathrm{E}=-2.17 \mathrm{~V}$ versus SCE [41] (which is harder to reduce than chloroform itself). If reduction proceeds through this pathway, the process stops there, as reduction of methylene chloride will not ordinarily occur. The radicals made in the first step of chloroform reduction may also recombine with each other, increasing the aliphatic chain length, but because reduction potential becomes more cathodic with the chain length of the hydrocarbon, the process will not self-accelerate. The reduction potential refers to the first one-electron step, but formal potentials for reduction of radicals are scarcely known. A reasonable assumption 
is that they are lower than those of the neutrals, so the process does not halt in the first step, but the particulars of reduction in chloroform-methanol mixtures, with $\mathrm{KCl}$ as supporting electrolyte, were not found during our earnest search effort. An educated guess, however, is that in a mixture of methanol and chloroform, chloroform is more susceptible to reduction, and methanol will be a proton donor.

1,4-Benzoquinone forms a reversible couple with its reduced form hydroquinone (standard potential $\mathrm{E}^{0}=$ $0.711 \mathrm{~V}$ \{versus $\mathrm{NHE}$, in water with $\mathrm{pH}=7$ ). This two-electron redox process is fast and reversible with the second electron transfer instantly following the first. Hydroquinone, the product of the second electron transfer, is in equilibrium with hydronium ions, making the whole process very sensitive to $\mathrm{pH}$; historically, an electrode of this type was even used as a $\mathrm{pH}$ indicator. In aprotic solvents, the second reduction step shifts to less positive potentials and becomes irreversible. The first polarographic reduction wave is rather unaffected by proton availability and is observed at about $\mathrm{E}_{1 / 2}=$ 0.1 (versus SCE) in acetonitrile or dimethylformamide, a value close to that obtained for water. 1,4-Benzoquinone's first reduction wave thus occurs at a potential significantly less negative than that of chloroform [34, 35].

For much of this work, details of electrochemical mechanisms are not essential, nonetheless it should not be ignored that electrochemical reduction or oxidation [41-43] can lead to a wide variety of released ions, which in turn, enter secondary chemical reactions, and also secondary electrochemical reactions. It is more common than not, that exotic secondary reaction pathways become possible. There are examples of reactions [41-43] which, from one substrate, produce dozens of different products. Of course, one also has to be cautious in transferring details of the reactions from one solvent to another, as well as from polarographic data acquired at a dropping mercury surface to other metals such as platinum, used here.

Specific conductivity of methanol is $1.510^{-9}\left[\Omega^{-1}\right.$ $\left.\mathrm{cm}^{-1}\right]$, conductivity of chloroform is at least 15 times less than that $[39,43]$. Those data refer to pure solvents; the solvents used in this experiment were not purified and the most important contaminant was water. Solvents used contain, according to manufacturer's specifications, $0.0013 \%$ of water (chloroform) and $0.0067 \%$ of water in methanol. Thus, a 50:50 mixture contains no less than $2.2 \mathrm{mM}$ of water.

\section{Appendix II}

\section{Estimation of Resistance of Electrolytic Cell}

Resistance between a small (micro) electrode and a large counter-electrode is practically defined by the properties of the small electrode. Total resistance is a function of specific resistance and geometrical parameters. For a simple geometry involving parallel planer electrodes, resis- tance is proportional to the distance between electrodes and inversely proportional to the area. Complex geometries require complicated formulas; often calculations can be done only by numerical simulation. For a microelectrode, it is argued that the small amount of volume in the vicinity of the surface contributes so much to the total resistance between electrodes that the rest can safely by ignored. The approximation works better as the electrode size gets smaller. It can be derived that electrolyte resistance in such a system is:

$$
\mathrm{R}=\mathrm{R}_{\mathrm{s}} / \mathrm{L}
$$

where $R_{s}$ is specific resistance (the inverse of specific conductivity), $\mathrm{L}$ is the circumference of the microelectrode.

For example, a $10 \mu \mathrm{M}$ solution of potassium chloride has a limiting molar conductivity $\Lambda_{0}=\left(\Lambda_{\mathrm{K}+}+\Lambda_{\mathrm{Cl}-}\right)=$ $150\left[\mathrm{~cm}^{2} / \Omega^{*} \mathrm{~mol}\right]$. Solution conductivity, $\sigma$, in a low concentration $(\mathrm{C})$ region, is a product of this value and concentration:

$$
\begin{aligned}
& \sigma=\Lambda * \mathrm{C}[1 / \Omega * \mathrm{~cm}] \\
& \sigma=150 * 50 * 10^{-9}=7.5 * 10^{-6}[1 / \Omega * \mathrm{~cm}] \\
& \mathrm{Rs}=1 / \sigma=133,333[\Omega * \mathrm{~cm}]
\end{aligned}
$$

our electrode has a diameter of $0.127 \mathrm{~mm}$, so the cicumference is $0.04 \mathrm{~cm}$

$$
\mathrm{R}=133,333 / 0.04=3.33 \mathrm{M} \Omega
$$

The same calculation for pure methanol with a specific conductivity of $1.5 \times 10^{-9}\left[1 / \Omega^{*} \mathrm{~cm}\right]$, gives a resistance $\mathrm{R}=16 \mathrm{G} \Omega$. This is a theoretical limit, of course, because in practice, methanol solutions contain water and residual salts which increase conductivity.

\section{Appendix III}

\section{Estimation of Field Strength Required for Cation Migration to Offset Velocity of Solution Flow}

Mobility of $\mathrm{K}^{+}$ion, in water, is $7.619^{*} 10^{-4} \mathrm{~cm}^{2} / \mathrm{sV}[22$, 44]. Flow of liquid in the capillary is $200 \mu \mathrm{l} / \mathrm{h}$. Assuming uniform flow through a cross section of $0.5 \mathrm{~mm}$, it gives a linear velocity in solution of $0.0283 \mathrm{~cm} / \mathrm{s}$. Poiseuille laminar flow is twice as fast in the center compared to average flow.

The velocity of migratory drift of an ion in the solution is a product of mobility and electric field, $\mathrm{v}=$ $u^{*} E$, where $u$ is mobility. From this we can get the field strength which corresponds to velocity of flow.

$$
\mathrm{E}=\mathrm{v} / \mathrm{u} \text {, which gives : } 0.0283 / 7.619 * 10^{-4}=37[\mathrm{~V} / \mathrm{cm}] \text {. }
$$

Such field strength is high. The derivative of our potential map is only about $5 \mathrm{~V} / \mathrm{cm}$, or less, in the region of positive values of $x$. 


\section{References}

1. Bailey, A. G. Electrostatic Spraying of Liquids; John Wiley and Sons, 1988; pp 1-193.

2. Blades, A. T.; Ikonomou, M. G.; Kebarle, P. Mechanism of Electrospray Mass Spectrometry. Electrospray as an Electrolysis Cell. Anal. Chem. 1991, 63, 2109-2114.

3. Juhasz, P.; Ikonomou, M. G.; Blades, A. T.; Kebarle, P. Electrospray, Mechanism, and Performance. In Methods and Mechanisms for Producing Ions from Large Molecules; Standing, K. G.; Ens, W. Eds.; Plenum Press: New York, 1991; pp 171-183.

4. Kebarle, P.; Ho, Y. On the Mechanism of Electrospray Mass Spectrometry. In Electrospray Ionization Mass Spectrometry; Cole, R. B., Ed.; John Wiley and Sons: New York, 1997; pp 3-63.

5. Van Berkel, G. J.; Zhou, F. Characterization of an Electrospray Ion Source as a Controlled-Current Electrolytic Cell. Anal. Chem. 1995, 67, 2916-2923.

6. Van Berkel, G. J. The Electrolytic Nature of Electrospray. In Electrospray Ionization Mass Spectrometry; Cole, R. B., Ed.; John Wiley and Sons: New York, 1997; pp 65-105.

7. Volk, K. J.; Yost, R. A.; Brajter-Toth, A. On-Line Electrochemistry/Thermospray/Tandem Mass Spectrometry, a New Approach to the Study of Redox Reactions: The Oxidation of Uric Acid. Anal. Chem. 1989, 61, 1709-1717.

8. Volk, K. J.; Yost, R. A.; Brajter-Toth, A. Electrochemistry On-Line with Mass Spectrometry. Anal. Chem. 1992, 64, 21A-33A.

9. Zhou, F.; Van Berkel, G. J. Electrochemistry Combined OnLine with Electrospray Mass Spectrometry. Anal. Chem. 1995, 67, 3643-3649.

10. Xu, X.; Lu, W.; Cole, R. B. On-Line Probe for Fast Electrochemistry/Electrospray Mass Spectrometry. Investigation of Polycyclic Aromatic Hydrocarbons. Anal. Chem. 1996, 68, 4244-4253.

11. Lu, W.; Xu, X.; Cole, R. B. On-Line Linear Sweep VoltammetryElectrospray Mass Spectrometry. Anal. Chem. 1997, 69, 24782484

12. McCarley, T. D.; Lufaso, M. W.; Curtin, L. S.; McCarley, R. L. Multiply Charged Redox-Active Oligomers in the Gas Phase: Electrolytic Electrospray Ionization Mass Spectrometry of Metallocenes. J. Phys. Chem. B 1998, 102, 10078-10086.

13. Deng, H.; Van Berkel, G. J. Electrochemical Polymerization of Aniline Investigated Using On-Line Electrochemistry/Electrospray Mass Spectrometry. Anal. Chem. 1999, 71, 4284-4293.

14. Mollah, S.; Pris, A. D.; Johnson. S. K.; Gwizdala, A. B., III; Houk, R. S. Identification of Metal Cations, Metal Complexes, and Anions by Electrospray Mass Spectrometry in the Negative Mode. Anal. Chem. 2000, 72, 985-991.

15. Levich, V. Physicochemical Hydrodynamics; Prentice Hall: Englewood Cliffs, NJ, 1962; pp 231-260.

16. Newman, J. Transport Processes in Electrolytic Solutions. In Advances in Electrochemistry and Electrochemical Engineering; Vol. V; Tobias, C. W., Ed.; Interscience Publishers: 1967; pp 87-136.

17. West, A. C.; Newman, J. Determination of Current Distributions Governed by Laplace's Equation. In Modern Aspects of Electrochemistry; Vol. XXIII; Conway, B. E.; Bockris, J. O'M.; White, R. E., Eds.; Plenum Press: New York, 1992; pp 101-148.

18. Van Berkel, G. J.; Giles, G. E.; Bullock, J. S., IV; Gray, L. J. Computational Simulation of Redox Reactions within a Metal Electrospray Emitter. Anal. Chem. 1999, 71, 5288-5296.

19. Li, Y.; Pozniak, B. P.; Cole, R. B. Mapping of Potential Gradients within the Electrospray Capillary. Anal. Chem. 2003, 75, 6987-6994.

20. Clopeau, M.; Prunet-Foch, B. Electrohydrodynamic Spraying Functioning Modes: A Critical Review. J. Aerosol Sci. 1994, 25, 1021-1036.

21. Pozniak, B. P.; Cole, R. B. Ambient Gas Influence on Electrospray Potential as Revealed by DEEP Mapping Within the ES
Capillary. Proceedings of the 52nd ASMS Conference on Mass Spectrometry and Allied Topics; Nashville, TN, May, 2004.

22. Vetter, K. J. Electrochemical Kinetics; Academic Press: New York, 1967; pp 1-40.

23. Parsons, R. Thermodynamic Methods for the Study of Interfacial Regions in Electrochemical Systems. In Comprehensive Treatise of Electrochemistry; Vol. I; Bockris, J. O'M; Conway, B, E; Yeager, E., Eds.; Plenum Press: New York, 1980; pp 1-42.

24. Trasatti, S. The Electrode Potential. In Comprehensive Treatise of Electrochemistry; Vol. I; Bockris, J. O'M; Conway, B, E; Yeager, E., Eds.; Plenum Press: New York, 1980; pp 45-82.

25. Bard, A. J.; Faulkner, L. R. Electrochemical Methods. Fundamentals and Applications; John Wiley and Sons: New York, 1980; pp $58-62$.

26. Tang, L.; Kebarle, P. Effect of the Conductivity of the Electrosprayed Solution on the Electrosprayed Current. Factors Determining Analyte Sensitivity in Electrospray Mass Spectrometry. Anal. Chem. 1991, 63, 2709-2715.

27. Tang, L.; Kebarle, P. Dependence of Ion Intensity in Electrospray Mass Spectrometry on the Concentration of the Analytes in the Electrosprayed Solution. Anal. Chem. 1993, 65, 3654-3668.

28. de la Mora, J. F.; Loscertales, I. G. The Current Emitted by Highly Conducting Taylor Cones. J. Fluid Mech. 1994, 260, 155-184.

29. Shtern, V.; Goldshtik, M.; Hussain, F. Generation of Swirl Due to Symmetry Breaking. Phys. Rev. E 1994, 49, 2881-2886.

30. Loscertales, I. G.; de la Mora, J. F. Experiments on the Kinetics of Field Evaporation of Small Ions from Droplets. J. Chem. Phys. 1995, 103, 5041-5060.

31. Barrero, A.; Gañán-Calvo, A. M.; Davila, J.; Palacio, A.; Gomez-Gonzalez, E. Low and High Reynold Number Flows Inside Taylor Cones. Phys. Rev. E 1998, 58, 7309-7314.

32. de la Mora, J. F.; Van Berkel, G. J.; Enke, C. G.; Cole, R. B.; Martinez-Sanchez, M.; Fenn, J. B. Electrochemical Processes in Electrospray Ionization Mass Spectrometry. J. Mass Spectrom. 2000, 35, 939-952.

33. Nicolis, G.; Portnow, J. Chemical Oscillations. Chem. Rev. 1973, 73, 365-384.

34. Kolthoff, I. M; Lingane, J. J. Polarography, 2nd ed.; Interscience: New York, 1952; pp 699-707

35. Janz, G. J.; Tomkins, R. P. T. Nonaqueous Electrolytes Handbook; Vol. II; Academic Press: New York, 1973; pp 527-603.

36. Van Berkel, G. J. Electrolytic Deposition of Metals on to the High Voltage Contact in an Electrospray Emitter: Implications for Gas-Phase Ion Formation. J. Mass Spectrom. 2000, 35, 773-783.

37. Van Berkel, G. J.; Zhou, F.; Aronson, J. T. Changes in Bulk Solution $\mathrm{pH}$ Caused by the Inherent Controlled-Current Electrolytic Process of an Electrospray Ion Source. Int. J. Mass Spec. Ion Processes 1997, 162, 55-67.

38. Van Berkel, G. J.; Asano, K. G.; Schnier, P. D. Electrochemical Processes in a Wire-in-a-Capillary Bulk-Loaded, Nano-Electrospray Emitter. J. Am. Soc. Mass Spectrom. 2001, 12, 853-862.

39. Strelow, H. Electrode Potentials in Non-Acqueous Solvents. . In The Chemistry of Non-Acqueous Solvents; Vol. I; Lagowski, J. J, Ed.; Academic Press: New York, 1966; p 129.

40. Brenner, A. Electrolysis of Non-Acqueous Systems. In Advances in Electrochemistry and Electrochemical Engineering; Vol V; Tobias, C. W., Ed.; Interscience Publishers: 1967; pp 205-248.

41. Fry, A. J. Synthetic Organic Electrochemistry; Harper and Row Publishers: New York, 1972; pp 170-187.

42. Popp, F. D.; Schultz, H. P. Electrolytic Reduction of Organic Compounds. Chem. Rev. 1962, 62, 19-40.

43. Weinberg, N. L.; Weinberg, H. R. Electrochemical Oxidation of Organic Compounds. Chem. Rev. 1968, 68, 449-523.

44. Sawyer, D. T.; Sobkowiak, A.; Roberts, J. L. Electrochemistry for Chemists, 2nd ed.; John Wiley and Sons: New York, 1995; p 318. 